\title{
Panorama des pratiques prescriptives dans les dictionnaires scolaires
}

\author{
Gasiglia, Nathalie \\ UMR 8163 (STL) du CNRS, Université Lille 3 \\ nathalie.gasiglia@univ-lille3.fr
}

\section{Introduction}

Cette contribution ${ }^{1}$ porte sur le rôle des dictionnaires dans l'apprentissage des normes langagières par les jeunes enfants scolarisés en France dans les cycles 2 et 3 de l'enseignement primaire (respectivement de la grande section de maternelle au cours élémentaire première année et du cours élémentaire deuxième année au cours moyen deuxième année). Elle prolonge quatre études connexes : dans la première, conduite avec Pierre Corbin (Corbin \& Gasiglia (2011)) et qui porte sur tous les types de marquages, l'examen des dictionnaires scolaires des trois dernières décennies a permis d'observer que la grande majorité des marquages ont également une fonction prescriptive, tandis que dans les suivantes (Gasiglia (2008a), $(2008 b)$ et $\left.(2008 c)^{2}\right)$, centrées sur les mots d'origine étrangère, les dépouillements ont permis de mettre en évidence que la mention de l'origine de certains emprunts peut être associée à des prescriptions de divers ordres.

La présente étude se concentre sur les dictionnaires scolaires imprimés destinés aux enfants de 5 à 12 ans publiés récemment ${ }^{3}$. Cette population particulière, qui construit progressivement sa compétence linguistique et s'initie, à l'école, à une pluralité de pratiques d'expression et de compréhension, apprend en même temps, à travers des répertoires en principe adaptés à son niveau, que les dictionnaires constituent des références que l'on consulte pour connaître les conventions de la société en matière d'usage du lexique, qu'il s'agisse de graphie, de prononciation, de sens ou de constructions, afin de reproduire ces conventions dans ses propres énoncés et de permettre ainsi à ceux-ci de s'inscrire harmonieusement et efficacement dans le flux des échanges verbaux. Au titre de cette acception large mais pertinente de la notion de prescription, qui, dans le cadre scolaire, ne fait qu'une place modeste au point de vue puriste, je me propose d'examiner successivement les prescriptions linguistiques qui concernent la forme des items et celles qui ont trait à leurs emplois, ainsi que les prescriptions non linguistiques qui trouvent à s'insérer dans les textes dictionnairiques. Il ne s'agira pas ici de couvrir extensivement la matière des dictionnaires pris en compte, mais de construire un cadre pour l'analyse d'une part des sélections macrostructurelles et microstructurelles opérées sur des critères normatifs et d'autre part des lieux textuels de prescription et des manières rédactionnelles associées, des plus explicitement injonctives à celles qui ne se donnent à lire que comme descriptives.

\section{Prescriptions concernant les formes des items}

Tout commence, à l'école, par l'apprivoisement des formes : savoir écrire ce qui se dit et lire ce qui est écrit $^{4}$, et, pour s'aider dans cette tâche, apprendre les conventions de structuration des dictionnaires et d'organisation de leurs articles, dont la maîtrise permettra d'accéder aux informations voulues sur les normes de graphie de tous les items et de leurs formes fléchies, et de prononciation de ceux ou celles ${ }^{5}$ pour lesquel(le)s elle peut être la plus problématique pour de jeunes apprenants. Certains composants des articles fournissent spécifiquement ces informations, mais celles-ci peuvent aussi apparaître, de façon indirecte et plus aléatoire, dans des composants dont la vocation première est d'une autre nature.

\subsection{Prescriptions explicites}

Les prescriptions explicites peuvent concerner aussi bien la graphie des items que leur prononciation. 


\subsubsection{Prescriptions concernant les formes graphiques}

L'une des pratiques en principe enseignées aux jeunes lecteurs est de prendre l'habitude d'exploiter les informations contenues dans leur dictionnaire afin de vérifier l'orthographe des mots qu'ils écrivent. Ce réflexe, que l'on peut commencer à inculquer au cycle 2, devrait être consolidé au cycle 3 et acquis à la fin de celui-ci. ${ }^{6}$ Dans les dictionnaires scolaires généraux, deux composants d'articles au moins doivent pouvoir être utilement consultés au titre de la vérification orthographique :

- Le composant d'identification ${ }^{7}$ fournit la forme dénominative (le lemme) des mots et communément une sélection de formes fléchies des noms et des adjectifs ${ }^{8}$, souvent écrites entièrement dans les dictionnaires pour le cycle 2 alors que certains ouvrages destinés au cycle 3 ne codent que la séquence finale modifiée (ex. : " chaud, chaude », "chasseur nom masculin 9 chasseuse nom féminin » et «normal, normale » dans le Larousse des débutants (cycle 2) vs «chaud, e », "chasseur, euse » et "normal, e, aux » dans le Larousse junior (cycle 3)) ${ }^{9}$. Telle que l'information orthographique est formulée, seul un apprentissage permet de l'exploiter et de choisir la forme graphique adéquate puis, en cas de flexions, de l'adapter aux contextes d'emplois effectifs. L'adresse ne fournit cependant jamais d'indications sur les formes verbales autres que l'infinitif: ce sont les modèles de conjugaison figurant dans les paratextes qui y pourvoient ${ }^{10}$, mais, alors qu'un renvoi des articles aux tableaux s'effectue au voisinage de l'adresse dans les dictionnaires pour le cycle 3 , ce n'est pas le cas dans ceux pour le cycle 2 .

- Les remarques orthographiques, présentes seulement dans certains articles, attirent l'attention des jeunes lecteurs sur plusieurs ordres de données :

- des règles d'orthographe flexionnelle (comme s.v. aveu dans le Larousse des débutants et le Larousse junior : « Au pluriel, on écrit des aveux. » / « Au pluriel : des aveux. »);

- des particularités d'accentuation (comme s.v. démêler dans le Larousse des débutants ou s.v. drôlerie dans Le Robert junior illustré depuis 2005 : «Le deuxième $e$ prend un accent circonflexe. » / « Attention à l'accent circonflexe du $\hat{o}$. $)$, ce qui vise à inciter les élèves à un effort régulier de vérification mais peut aussi contribuer à prévenir de possibles erreurs, si ces remarques sont lues à l'occasion d'une autre recherche d'information ;

- dans le Dictionnaire Hachette Benjamin, des renvois vers les tableaux de conjugaison figurant dans les paratextes, à partir des seuls articles consacrés à des verbes conjugués dans les tableaux ;

- des variantes orthographiques, ce qui est fait en validant explicitement des formes concurrentes ${ }^{11}$ (comme s.v. clé dans Le Robert benjamin et Le Robert junior illustré : «Tu peux écrire aussi clef.» / " On peut écrire aussi clef [kle]. » jusqu'en 2010, « On écrit parfois clef [kle]. » en 2013) ou en expliquant les règles d'emploi des allomorphes (s.v. de dans le Larousse des débutants, en conjonction avec des indications phoniques et grammaticales pas strictement prescriptives : «De s'écrit $d$ ' devant une voyelle ou un « $\mathrm{h}$ » muet : le livre d'Anaïs. On ne dit pas « de le » ni « de les» mais $d u$ et des: le livre du professeur, des élèves. ») ou encore en justifiant l'existence des variantes par des raisons étymologiques (s.v. cari dans le Dictionnaire Hachette junior : « On écrit aussi cary, ou curry, comme en anglais. »);

- des mises en garde relatives à des confusions lexicales, qui peuvent concerner des mots homonymes ou paronymes, comme respectivement, dans le Larousse junior, "Ne confonds pas avec la foi ou une fois. » (s.v. foie) et « Ne confonds pas avec mille (1). » (s.v. mile).

\subsubsection{Prescriptions concernant les formes phoniques}

La recherche d'une forme phonique d'un mot peut être motivée par une difficulté de lecture. Cette démarche suppose que les enfants s'accoutument à décoder des transcriptions phonétiques en Alphabet Phonétique International par le canal de méthodes d'apprentissage de la lecture associant les sons à leurs différentes graphies possibles : si c'est le cas, ils peuvent être à même de prendre l'habitude de consulter leur dictionnaire pour oraliser un mot qu'ils ne savent pas lire, soit qu'ils ne le connaissent pas, soit qu'ils ne l'identifient pas par sa forme graphique ${ }^{12}$. Ici encore plusieurs composants d'articles peuvent soutenir leurs efforts :

- Le composant de prononciation, sélectivement présent dans des articles du Dictionnaire Hachette benjamin pour le cycle 2, du Dictionnaire Hachette junior et du Robert junior illustré pour le cycle 3, fournit 
une transcription du lemme des mots décrits et éventuellement aussi celle de formes fléchies (comme celle de bœufs s.v. bœuf dans le Dictionnaire Hachette benjamin et le Dictionnaire Hachette junior ${ }^{13}$ ou celle de yeux s.v. oil dans le premier $\left.{ }^{14}\right)^{15}$. Cette information peut éventuellement être associée à une donnée d'une autre nature, en particulier une information étymologique, pour des emprunts qui ne respectent pas totalement les règles phonographiques du français comme zoom ${ }^{16}$. Comme pour la forme orthographique, le consultant doit généralement faire un travail d'adaptation de la ou des forme(s) phonique(s) fournie(s), afin, par exemple, de faire les liaisons imposées par le contexte qui a motivé la consultation du dictionnaire. Toutefois, quand un item a des variantes régulières et irrégulières distributionnellement contraintes, le composant de prononciation peut expliciter leurs emplois, comme pour [di], [dis] ou [diz] s.v. dix dans le Dictionnaire Hachette junior : «On prononce [dis] quand dix est employé seul, [di] devant une consonne ou un $\mathrm{h}$ aspiré, [diz] devant une voyelle ou un $\mathrm{h}$ muet. ».

- Le composant orthographique peut lui aussi prendre en charge la présentation d'une transcription phonétique de forme fléchie, qui n'est alors présentée qu'en son sein (comme celle de boufs s.v. bœuf dans Le Robert junior illustré ou celle de yeux s.v. œil dans le Dictionnaire Hachette junior : « On dit un bæuf [bœf], des bœufs [bø]. » / « Pluriel : des yeux [jø]. »).

- Les composants de remarques polyvalents qui fournissent des informations phonographiques visent souvent à prévenir des fautes :

- certains attirent explicitement l'attention sur les difficultés de lecture prévues par les lexicographes et ciblent généralement un segment problématique dans la forme dénominative du mot (comme pour les noms en -tie dans le Larousse des débutants ou le Larousse junior (en 2003 mais plus depuis 2008), par exemple s.v. acrobatie : «On écrit tie mais on prononce [si]. »);

- d'autres motivent le fait de présenter la transcription d'un item par son étymologie (comme dans le Larousse junior, s.v. zoom : " C'est un mot anglais, on prononce [zum]. ») ;

- d'autres introduisent des variantes de prononciation libres (comme [zo] ou [zoo] s.v. zoo dans le Larousse des débutants et le Larousse junior : «On prononce [zo] ou [zoo]. ») ou contraintes (comme [di], [dis] ou [diz] s.v. dix dans le Fleurus junior : « On prononce [dis] lorsqu'on emploie le mot seul, [di] devant une consonne ou un « $\mathrm{h}$ » aspiré et [diz] devant une voyelle ou un « $\mathrm{h} »$ muet. »);

- d'autres encore tentent de mettre en garde au sujet de prononciations observées mais jugées fautives (le Larousse junior signale par exemple que le $p$ de dompter ne devrait pas être prononcé : «On ne prononce pas le p. ») : seul ce dernier cas relève de la prescription considérée dans son acception la plus puriste.

\subsection{Prescriptions implicites}

Les prescriptions de forme implicites ne peuvent concerner que les formes graphiques des items décrits dans les dictionnaires imprimés. Elles peuvent être véhiculées par les définitions et par les contextualisations.

\subsubsection{Prescriptions dans les définitions}

Il arrive, marginalement, que des définitions fournissent tacitement une information flexionnelle sur l'item défini. Ce fait se rencontre dans un petit nombre d'articles de dictionnaires qui recourent à des schémas définitionnels de forme " $X$ est $Y$ " ou " $X$, c'est $Y$ ", dans lesquels $X$ reprend l'item de l'adresse, ordinairement sous sa forme canonique (comme $s . v$. éboueur dans le Larousse des débutants ${ }^{17}$ ), mais occasionnellement sous une forme fléchie : ainsi en va-t-il dans le Larousse des débutants s.v. aveugle, sourd, sourde ou écaille ${ }^{18}$, et dans Le Robert benjamin s.v. écaille $1 .^{19}$. Les données de ce type sont cependant trop rares pour qu'il faille y voir la mise en œuvre d'un principe d'exploitation du module définitionnel à des fins flexionnelles : il ne s'agit que d'exceptions motivées probablement par l'appréciation de conditions d'usage particulières des mots concernés.

\subsubsection{Prescriptions dans les contextualisations}

La fonction des contextualisations étant de montrer la manière dont les mots décrits s'emploient, il est naturel qu'il en aille autrement que pour les définitions, et qu'elles soient un lieu privilégié pour en présenter 
différentes formes graphiques. Elles sont notamment utilisées de façon assez récurrente pour afficher des formes de noms ou d'adjectifs fléchies au pluriel, sans que la gestion de la redondance ou de la complémentarité avec d'autres composants des articles pouvant apporter les mêmes informations soit nécessairement homogène et méthodique : ainsi, dans le Dictionnaire Hachette benjamin, qui a pour caractéristique de ne présenter dans le composant d'adressage que le lemme des noms et adjectifs variables (cf. n. 8), la contextualisation peut, selon les cas,

- montrer à elle seule une des formes fléchies (comme le féminin s.v. infini ou le pluriel s.v. indiscipliné : «On ne peut pas compter les étoiles : il y en a une quantité infinie. » / «Certains élèves de cette classe sont indisciplinés. »),

- donner à voir celle qui n'est pas prise en charge par une remarque spécifique (comme le féminin singulier s.v. infernal ou normal : "La chaleur a été infernale pendant tout le voyage. » ou "Xavier n'a pas de fièvre, sa température est normale. » en contextualisation / « Des fillettes infernales, des enfants infernaux. » ou en remarque, cf. n. 8),

- ou présenter la même forme que celle de la remarque (comme le féminin s.v. individuel : «Dans cette classe, chaque enfant a une table individuelle. » en contextualisation / «Un billet individuel, une chambre individuelle. » en remarque) ou que la légende d'une iconographie (comme le pluriel s.v. lointain : «Thomas rêve de voyager dans des pays lointains. » en contextualisation / «On trouve des huttes dans certains pays lointains. » en légende). ${ }^{20}$

\section{Prescriptions concernant les emplois des items}

Tout comme les prescriptions afférentes aux formes graphiques et phoniques des items, celles qui concernent diverses modalités de leurs emplois peuvent aussi être explicitées dans des rubriques spécifiques ou être suggérées de façon plus diffuse dans des composants dédiés foncièrement à la transmission d'autres types d'informations.

\subsection{Prescriptions explicites}

Les prescriptions explicites peuvent avoir pour objet la combinatoire des items ou la variation socioénonciative des usages lexicaux.

\subsubsection{Prescriptions concernant la combinatoire lexicale}

La sélection des arguments (sujet ou complément(s)) des verbes peut occasionnellement faire l'objet de prescriptions normatives explicites comme celles que l'on observe dans les remarques présentes s.v. emmener et emporter dans le Larousse des débutants ( On dit «j'ai emmené une personne» mais «j'ai emporté un objet ».»/ « On dit « il a emporté un objet» mais « il a emmené une personne ».») ou s.v. emmener dans le Dictionnaire Hachette junior (« On emmène une personne et on emporte un objet. »).

\subsubsection{Prescriptions concernant la variation socioénonciative des usages lexicaux}

Trois composants d'articles (les marques, les définitions et les remarques) peuvent être exploités, chacun avec des principes rédactionnels spécifiques, afin de fournir aux jeunes consultants des indications leur permettant de repérer l'emploi d'un mot dans le temps ou dans l'espace et de les informer sur des conventions d'usage de divers ordres. La sélection de marquages présentée ci-dessous, qui adopte un principe de classement inspiré de Hausmann (1977, chap. 8, et 1989), illustre la diversité de ces prescriptions explicites. 


\section{1) Variations socioénonciatives décrites par une marque}

Composant usuellement exploité pour les marquages socioénonciatifs, les marques ne sont pas absentes des dictionnaires scolaires, même si elles y sont plutôt mentionnées sous forme non abrégée ou qu'elles y sont représentées par des icônes. Elles peuvent fournir des prescriptions

- diachroniques ${ }^{21}$, comme s.v. aliéné, e et besogne dans le Larousse junior : « Mot ancien »;

- diaévaluatives ${ }^{22}$, comme s.v. ramassis dans le Larousse junior : « Mot péjoratif. »;

- diaphasiques ${ }^{23}$, comme s.v. jugeote et kif-kif dans le Larousse junior (« Mot familier. ») ou comme s.v. zouave 2., dans le même ouvrage, où l'adresse microstructurelle «Faire le zouave», qui représente l'item polylexical décrit dans cette subdivision d'article, est précédée par la marque «(Familier). »;

- ou encore diatextuelles ${ }^{24}$ exprimées,

- soit textuellement comme s.v. omettre, omission et omnipotent dans le Larousse junior : « Mot littéraire. »; - soit, dans le Larousse des débutants jusqu'en 2010, au moyen d'une icône qui figurait un livre-papillon, qui était placée en début d'article (par exemple s.v. demeure et licorne) et qui indiquait que le mot concerné, pour au moins un de ses emplois, se rencontrait «dans un conte, une chanson » ("Comment utiliser le Larousse des débutants », p. VI en 2005 / p. 6 en 2010) ${ }^{25}$.

\section{2) Variations socioénonciatives décrites dans les définitions}

Les prescriptions socioénonciatives qui figurent dans les définitions sont relativement peu nombreuses. Elles y sont formulées en articulation avec les descriptions sémantiques et concernent des variations

- diachroniques, comme s.v. batracien dans le Larousse junior : « Nom donné autrefois aux amphibiens. »; - diaphasiques, comme s.v. joujou dans le Larousse junior («Jouet, dans le langage des enfants.») ou s.v. gamin, ine dans le Dictionnaire Hachette junior («Synonyme familier d'enfant. ») ;

- ou encore diatextuelles, comme s.v. licorne dans Mon premier Bescherelle illustré («Dans les légendes, la licorne a une longue corne au milieu du front. ») ou s.v. azur dans le Larousse des débutants («En poésie, l'azur est la couleur bleue du ciel. »).

\section{3) Variations socioénonciatives décrites dans les remarques}

Outre les recommandations proprement linguistiques qu'elles comportent (cf. § 2.1), les remarques constituent le lieu dominant du traitement des informations socioénonciatives. Les prescriptions explicites sont de natures plus variées que dans les composants précédents, elles nécessitent éventuellement un certain travail d'interprétation de la part des jeunes consultants concernant la portée de ce qui leur est indiqué et elles peuvent être d'ordre

- diachronique, comme s.v. domestique dans le Larousse des débutants et Le Robert benjamin: «Aujourd'hui, on dit un employé de maison, une employée de maison.» / " Aujourd'hui, on dit plutôt employé de maison. »;

- diaévaluatif, comme s.v. gueule dans le Larousse junior : «Gueule est un mot grossier quand on parle [de la bouche ou du visage] d'une personne. $»^{26}$;

- diamédial ${ }^{27}$, comme s.v. flot dans Le Robert benjamin : «C'est un mot que l'on ne dit pas beaucoup, on le rencontre surtout dans les livres. »;

- dia-intégratif ${ }^{28}$, pour déconseiller l'emploi d'anglicismes, comme s.v. cameraman dans le Larousse junior de 2008 ( «C'est un mot anglais, il vaut mieux dire cadreur, cadreuse. ») ${ }^{29}$, ou signifier leur proscription dans certaines aires francophones, comme s.v. week-end dans le Dictionnaire Hachette junior («Week-end est un mot anglais [...] On n'emploie pas ce mot au Québec : on dit « fin de semaine ».»); 
- dianormatif ${ }^{30}$

- pour indiquer une dénomination jugée plus adéquate, comme s.v. fou 1. dans Le Robert junior illustré de 2013 : «On dit plutôt malade mental » ${ }^{31}$;

- ou pour signaler un manque d'adéquation référentielle, comme s.v. gâchette dans Le Robert benjamin:

« On dit souvent « appuyer sur la gâchette », mais on a tort. Il faut dire « appuyer sur la détente. » »;

- diaphasique, comme s.v. gamin, gamine dans Le Robert benjamin de 2013 ( « Gamin et gamine sont des mots familiers. On peut dire un petit garçon ou une petite fille. $\rangle^{32}$ ) ou $s . v$. joujou et kinésithérapeute dans Le Robert junior illustré depuis 2005 («On utilise ce mot quand on s'adresse à de très jeunes enfants. ${ }^{33}$ et «On dit familièrement kiné. ») ou encore s.v. kinésithérapeute dans le Larousse junior («On emploie souvent l'abréviation familière kiné. ») ;

- diatopique ${ }^{34}$, comme s.v. wassingue dans Le Robert junior illustré : « Ce mot est utilisé dans le nord de la France et en Belgique. »;

- ou encore diatextuel, comme s.v. châtier dans Le Robert junior illustré de 2005 à 2010 : «Ce mot est littéraire. $\gg{ }^{35}$.

\subsection{Prescriptions implicites}

Aux prescriptions explicites s'ajoutent celles, implicites, que supposent certaines omissions d'items ou d'emplois ou qu'impliquent telles définitions ou contextualisations.

\subsubsection{Prescriptions par la non-description d'un item ou d'un emploi}

Les omissions d'items ou d'emplois peuvent être motivées par le souhait de mieux valoriser ceux dont l'usage est recommandable et qui sont, eux, présentés, mais elles peuvent également être motivées par le souhait de faire de la place pour d'autres descriptions ou par celui de marquer un désintérêt assumé pour ce qui est exclu.

\section{1) Non-sélection d'items ou d'emplois usités valorisant des concurrents recommandés}

Une forme particulière et probablement mineure de prescription indirecte peut consister à ne pas faire figurer dans le dictionnaire des items usités mais en concurrence dans l'usage avec des items que leur préfèrent les puristes : ainsi en va-t-il, par exemple, du verbe indifférer (Cela m'indiffère que $P$ / (Que) P(, cela) m'indiffère), dont le Bescherelle pratique rappelle la mise en garde dont il fait volontiers l'objet et les expressions qui lui sont préférées ${ }^{36}$, et qui est absent de l'ensemble des dictionnaires actuels pour le cycle 3 , lesquels, par contre, à l'exception du Dictionnaire Maxi débutants, font tous mention de l'emploi d'indifférent avec un argument propositionnel souvent associé à une dislocation (Cela m'est indifférent que P / (Que) P(, cela) m'est indifférent $)^{37}$.

Une variante de cette manière de procéder consiste dans l'omission d'un emploi réprouvé d'un item donné au bénéfice de son emploi approuvé : les dictionnaires pour le cycle 3 qui ont un article alternative procèdent de cette façon en n'évoquant, pour définir ce mot, que la notion de choix entre deux options, sans faire mention de son usage répandu pour référer à l'une de celles-ci ${ }^{38}$, en conformité tacite, ici encore, avec la prescription explicitement exprimée dans le Bescherelle pratique $e^{39} .40$

\section{2) Non-sélection d'items ou d'emplois sans valorisation de concurrents}

Les dictionnaires scolaires présentent une sélection relativement limitée d'items (environ 6000 à 8000 pour le cycle 2 et 20000 à 25000 pour le cycle 3) et, pour chacun de ceux qui sont polysémiques, une sélection d'emplois qui se concentre sur ce qui est utile dans le cadre scolaire ou qui enrichira utilement la compétence langagière des consultants, comme le rappelle, par exemple, Rey (2001: XXVI) : " ces nomenclatures de dictionnaires ne coïncident pas avec l'usage réel des enfants auxquels ils s'adressent : de nombreux mots qui y figurent sont pratiquement lettre morte dans le discours spontané ; d'autres, d'un emploi fré- 
quent - les "mots de la tribu", famille, lycée, etc., et bien entendu les "gros mots" et termes à la mode sont pour partie exclus des nomenclatures. ». Un dépouillement systématique des nomenclatures et des descriptions sémantiques proposées par les dictionnaires étudiés permettait de mieux évaluer la part de cette prescription silencieuse.

\subsubsection{Prescriptions dans les définitions}

Il peut aussi advenir que des prescriptions implicites se décèlent dans certaines définitions. Ainsi en va-til par exemple, dans le Fleurus junior et le Dictionnaire Auzou junior, de celles d'emmener (respectivement « Amener une personne avec soi d'un lieu à un autre. » et « Conduire une personne d'un lieu à un autre. »), qui prennent en charge la restriction normative de la sélection argumentale à des substantifs référant à des humains évoquée au $§ 3.1 .1$. Pour le même verbe, dans le Larousse des débutants et le Dictionnaire Hachette junior, la définition annonce implicitement l'information explicitée dans la remarque qui suit : « Emmener une personne, c'est la prendre avec soi pour aller quelque part. » dans le premier et « Amener quelqu'un avec soi. » dans le second.

\subsubsection{Prescriptions dans les contextualisations}

Les contextualisations jouent aussi un certain rôle comme vecteurs de normes d'usage montrées sans être expressément formulées. Elles sont notamment utilisées pour afficher :

- des prescriptions syntaxiques, comme celle concernant la rection directe du verbe pallier dans la majorité des dictionnaires pour le cycle 3 qui le répertorient ${ }^{41}$, qui ne reçoit le soutien explicite d'une remarque normative que dans Le Robert junior illustré depuis 2005 et le Dictionnaire Maxi débutants ${ }^{42}$, ou encore comme le choix de la préposition chez préférentiellement à à dans aller chez le coiffeur, qui motive celui de la contextualisation illustrant les articles coiffeur, euse des dictionnaires pour le cycle 3 qui présentent cet item en contexte ${ }^{43}$

- des indications relatives aux accords entre sujets et verbes, comme dans Le Robert junior illustré, où les sujets féminins des contextualisations des formes pronominales se blesser et se casser ("Léa s'est blessée à la main »s.v. blesser 1. depuis $2005^{44}$ et «Elle s'est cassé la jambe en faisant du ski » s.v. casser 2.) montrent à qui sait les voir le comportement différent des verbes et l'incidence de celui-ci sur la graphie de leur participe ${ }^{45}$.

\section{Prescriptions comportementales}

Composants multifonctionnels par essence des articles de dictionnaires, les contextualisations constituent des espaces d'expression qui peuvent aussi accueillir des prescriptions non linguistiques de divers ordres, comme, par exemple,

- des principes éducatifs, qui peuvent être exprimés soit comme ils le seraient lors d'un échange verbal familial (comme, dans le Larousse des débutants, s.v. 2. avant 1 : «Tu pourras aller jouer, mais finis ton repas avant. »), soit de manière plus explicitement prescriptive (comme $s . v$. bâiller, dans le même dictionnaire, ou s.v. 1. avant 4 dans le Larousse junior : " On met sa main devant sa bouche quand on bâille. »/ «Prière de frapper avant d'entrer. »);

- des règles de conduite juridiquement encadrées, comme s.v. amende, contravention ou encore contrefaçon dans le Larousse junior : "Elle a eu une amende car elle n'avait pas attaché sa ceinture. " / "Avoir une contravention pour excès de vitesse. » / « La contrefaçon de billets de banque est punie par la loi. »;

- des prescriptions de conduites valorisées, dans le même dictionnaire, exprimées sous forme de relation causale, comme s.v. sécurité ( Respecter les consignes de sécurité [permet d'éviter les accidents]. ${ }^{46}$ ), ou en soutenant un point de vue, comme s.v. avantage ( L'avantage du vélo, c'est qu'il ne pollue pas. »); 
- mais aussi, toujours dans le Larousse junior, des prescriptions d'hygiène de vie, factuelles et assertives, comme s.v. fumer ( "Fumer est dangereux pour la santé. »), ou exprimées de manière injonctive, comme s.v. sel ou sucrerie («Il faut goûter un aliment avant d'y ajouter du sel. ${ }^{47} /$ «Ne mangez pas trop de sucreries. »), ou encore prenant la forme anodine d'énoncés narratifs, susceptibles cependant d'induire chez les enfants une réaction orientée axiologiquement, comme s.v. grignoter («Elle grignote des sucreries toute la journée. »).

\section{Conclusion}

Pour chacun "le dictionnaire" est un ouvrage de référence, un partenaire dans l'apprentissage du "bon" usage de notre langue. Pour certains lexicographes, "le dictionnaire" est également un ouvrage dont l'une des missions consiste à aider les enfants à devenir des personnes capables de trouver leur place dans des échanges codifiés (riches en conventions verbales et sociales) et capables de situer leur pratique langagière dans le temps et dans l'espace. L'étude menée ici a permis de dégager les types de prescriptions linguistiques, socioénonciatives et comportementales que peuvent formuler les dictionnaires actuels destinés aux élèves des cycles 2 et 3 de l'enseignement primaire et les moyens qu'ils sont susceptibles de mettre en œuvre à cette fin, mais de manière transversale, sans s'attacher à dégager d'éventuelles spécificités de dictionnaires particuliers. Dans de possibles développements ultérieurs de la recherche, en prenant appui sur le cadre d'analyse qui a été mis en place, il serait utile de comparer plus en détail les options descriptives des répertoires considérés, d'établir systématiquement ce qui motive que certains items ou emplois soient exclus des nomenclatures ou qu'ils fassent l'objet d'indications à orientation prescriptive, d'évaluer si celles-ci sont régulièrement présentes dans les dictionnaires scolaires, si elles sont aisément décodables par les consultants auxquels elles s'adressent et si, au sein de chaque ouvrage, elles sont bien mentionnées pour tous les items qui partagent certaines propriétés.

\section{Références bibliographiques}

\section{Répertoires métalinguistiques}

Bescherelle pratique $=$ Kannas, C. (2003).

Dictionnaire Auzou junior. (2006). Paris : Éditions Philippe Auzou ; nouv. tir. (2011).

Dictionnaire des mots difficiles à lire = Presse, N. (2001).

Dictionnaire Hachette benjamin. (2011). Paris : Hachette Éducation.

Dictionnaire Hachette junior. (2010). Paris : Hachette Éducation.

Dictionnaire Maxi débutants CE1-CE2-CM1-CM2. (1999). Paris : Larousse.

Fleurus junior. Dictionnaire encyclopédique. (2004). Paris : Éditions Fleurus.

Grinevald, F. (2007). Les mots difficiles à écrire. Paris : Éditions Rue des Écoles; version téléchargeable (URL valide le 15/03/2014) : http://www.maif.fr/enseignants/solutions-educatives/apprentissage/mots-difficiles-a-ecrire.html.

Kannas, C. (2003). Le Bescherelle pratique de la langue française. Paris : Hatier.

Kannas, C. (2007). Mon premier Bescherelle illustré. Paris : Hatier.

Larousse des débutants. (2005). Paris : Larousse ; nouv. tir. (2010); nouv. tir. révisé (2011).

Larousse junior. (2003). Paris : Larousse ; nouv. éd., Dictionnaire junior. (2008) ; nouv. tir. révisé (2013).

Le Grand dictionnaire de français. (2004). [ville non indiquée] : Éclairs de Plume.

Le Robert benjamin. (2005). Paris : Dictionnaires Le Robert ; nouv. tir. (2012) ; nouv. éd. (2013).

Le Robert junior illustré. (1993). Paris : Dictionnaires Le Robert ; nouv. tir. révisé (2003); nouv. éd. (2005) ; nouv. tir. (2010) ; nouv. éd. (2013). 
Les mots difficiles à écrire = Grinevald, F. (2007).

Mon grand dictionnaire illustré. (2006). [ville non indiquée] : Cerf Volant / Éclairs de Plume.

Mon premier Bescherelle illustré = Kannas, C. (2007).

Presse, N. (2001). Dictionnaire des mots difficiles à lire. Paris : Éditions Garnier / Rue des Écoles; nouv. tir., Rue des Écoles. (2004). (2007) ; version téléchargeable (URL valide le 15/03/2014) : http://www.maif.fr/enseignants/ solutions-educatives/apprentissage/mots-difficiles-a-lire.html?xtmc=dictionnaire_des_mots_difficiles_a_ecrire \&xtcr=4.

\section{Autres références}

Corbin, P. (2004). Théorie et pratique de la formation professionnelle de lexicographes : treize ans d'expérience lilloise. In G. Williams \& S. Vessier (eds), Proceedings of the Eleventh EURALEX International Congress, EURALEX 2004, Lorient, France, July 6-10, 2004, vol. 1, Université de Bretagne-Sud. 13-35 [éd. fautive] ; rééd. conforme au texte original In P. Corbin, Avec des dictionnaires pour compagnons, habilitation à diriger des recherches, vol. 3, Villeneuve d'Ascq : Université Charles-de-Gaulle - Lille 3 / L'Atelier C. 1161-1186.

Corbin, P. \& Gasiglia, N. (2011). Éléments pour un état de la description de la variété des usages lexicaux dans les dictionnaires français monolingues (1980-2008). In F. Baider, E. Lamprou \& M. Monvielle-Burston, La marque lexicographique. États présents, voies d'avenir, Limoges : Lambert-Lucas. 17-37.

Gasiglia, N. (2008a). Le traitement des emprunts dans les dictionnaires d'apprentissage français : options descriptives et choix rédactionnels. In J. Pruvost dir., Les Journées des dictionnaires de Cergy. Dictionnaires et mots voyageurs. Les 40 ans du Petit Robert, coll. Actes de colloque, Eragny-sur-Oise : Éditions des Silves. 153-212.

Gasiglia, N. (2008b). Le traitement des anglicismes dans quelques dictionnaires français pour jeunes lecteurs. In F. Maniez \& P. Dury dir., Lexicographie et terminologie : histoire de mots. Hommage à Henri Béjoint, Travaux du CRTT, Gap : Louis Jean Imprimeur. 157-174.

Gasiglia, N. (2008c). Description of loan words in French school dictionaries: treatment of words of foreign origin in Dictionnaire Hachette junior (2006) and Le Robert junior illustré (2005). In E. Bernal \& J. DeCesaris eds, Proceedings of the Thirteenth EURALEX International Congress, EURALEX 2008 (Barcelona 15-19 july 2008), Sèrie Activitats 20, Barcelone : Institut Universitari de Lingüística Aplicada. 1115-1122.

Hausmann, F.J. (1977). Einführung in die Benutzung der neufranzösischen Wörterbücher. Romanistische Arbeitshefte 19. Tübingen : Max Niemeyer Verlag.

Hausmann, F.J. (1989). Die Markierung im allgemeinen einsprachigen Wörterbuch: eine Übersicht. In F.J. Hausmann, O. Reichmann, H.E. Wiegand \& L. Zgusta Hrsg., t. 1, art. 53. 649-657.

Hausmann, F.J., Reichmann, O., Wiegand, H.E. \& Zgusta L. Hrsg. (1989-1991). Wörterbücher / Dictionaries / Dictionnaires. Ein internationales Handbuch zur Lexikographie / An International Encyclopedia of Lexicography / Encyclopédie internationale de lexicographie, 3 vol., Berlin / New York : Walter de Gruyter.

Hausmann, F.J. \& Wiegand, H.E. (1989). Component parts and structures of general monolingual dictionaries: a survey. In F.J. Hausmann, O. Reichmann, H.E. Wiegand \& L. Zgusta Hrsg., t. 1, art. 36. 328-360.

Qu'apprend-on à l'école élémentaire? Les nouveaux programmes. (2002). Paris : Centre National de Documentation Pédagogique / XO Éditions.

Rey, A. (2001). Préface du Grand Robert de la langue française. In Le Grand Robert de la langue française. Dictionnaire alphabétique et analogique de la langue française, nouv. éd. augmentée de la $2^{\mathrm{e}}$ éd., vol. 1, Paris : Dictionnaires Le Robert. XIX-LV.

\footnotetext{
${ }^{1}$ Merci à Pierre Corbin et Alise Lehmann, qui, par leur relecture critique de cet article, ont contribué à l'enrichissement de mon propos. Merci également aux relecteurs anonymes pour leurs retours constructifs.

${ }^{2}$ Ces trois articles présentent respectivement :

- pour Gasiglia (2008a), un panorama des modalités de traitement des indications d'origines étrangères des mots empruntés ou hérités établi à partir du dépouillement d'une large sélection d'ouvrages scolaires, ou d'orientation plus culturelle ou ludique destinés aux jeunes lecteurs ;
} 
- pour Gasiglia (2008b), une étude des liens entre mentions d'origine anglaise ou anglo-américaine et difficultés phonographiques décrites à propos des items dans les dictionnaires scolaires ;

- pour Gasiglia (2008c), une analyse des différentes informations fournies à propos des mots empruntés dans deux dictionnaires scolaires destinés aux élèves du cycle 3 (le Dictionnaire Hachette junior (2006) et Le Robert junior illustré (2005)).

${ }^{3}$ Les mises en forme des citations d'articles sont aussi approchées que possible de celles des dernières éditions des dictionnaires cités.

${ }^{4}$ Parmi les compétences devant être acquises en fin de cycle 2 (cf. Qu'apprend-on à l'école élémentaire?,p. 94) :

« Être capable de :

- proposer une écriture possible (et phonétiquement correcte) pour un mot régulier,

- déchiffrer un mot que l'on ne connaît pas,

- identifier instantanément la plupart des mots courts (jusqu'à quatre ou cinq lettres) et les mots longs les plus fréquents.

$[\ldots]$

- orthographier la plupart des "petits mots" fréquents (articles, prépositions, conjonctions, adverbes...),

- écrire la plupart des mots en respectant les caractéristiques phoniques du codage ».

Voir également les instructions du Ministère de l'éducation nationale consultables en ligne (URL valide le 15/03/2014) : http://www.education.gouv.fr/bo/2002/hs1/cycle2.htm.

${ }^{5}$ Dans le Dictionnaire Hachette benjamin, par exemple, cent trente-six lemmes sont transcrits mais aussi quatre formes fléchies (bœufs, œufs, os (au pluriel) et yeux, cf. § 2.1.2).

${ }^{6}$ En classe ce sont usuellement des dictionnaires scolaires généraux qui sont utilisés, mais certains éditeurs produisent également des dictionnaires thématiques. Parmi eux, Les mots difficiles à écrire, paru en 2007 et destiné aux élèves de l'école élémentaire, compte un millier d'articles de facture classique qui sont distribués dans trente rubriques thématiques ( «Les accents »; «Le tréma »; «La lettre $h » ; ~ «$ Le $m$ devant $m, b, p » ;$ «Les consonnes doublées »; etc.) et qui sont précédés, au sein de chaque rubrique, d'un exposé de la règle qui la motive, précédé lui-même d'un texte riche en mots difficiles du point de vue de cette règle. Cette nomenclature donne une certaine vue du lexique dont la graphie présente les types de difficultés relevés, mais le nombre d'items décrits semble être plus déterminé en fonction de celui retenu pour le Dictionnaire des mots difficiles à lire destiné au même public dès 2001 (cf. n. 12) qu'en fonction du nombre d'items pertinents pour le public cible et présentant les difficultés orthographiques décrites.

${ }^{7}$ C'est le nom de la zone prédéfinitionnelle des articles dans la terminologie adoptée par Hausmann \& Wiegand (1989: 341).

${ }^{8}$ Ce n'est toutefois pas le cas dans le Dictionnaire Hachette benjamin, qui ne présente que la forme dénominative en adresse et qui n'utilise que les remarques pour fournir, sélectivement, cette information flexionnelle de façon explicite (s.v. normal : "Des analyses normales, des bruits normaux. ») et les contextualisations pour la donner à voir implicitement, cf. infra $\S 2.2 .2$.

${ }^{9}$ Dans Le grand dictionnaire de français (cycle 3), les informations relatives aux formes fléchies sont présentées dans le composant d'adressage quand la mise au féminin consiste à concaténer un $e$ final au masculin, et dans le composant flexionnel dans les autres cas : « chaud(e)», «chasseur : n. (f. chasseuse)» (en adresse d'article subordonné s.v. chasser) et «normal(e) : [...] 1-adj. (plur. normaux)». Le répertoire correspondant pour le cycle 2, Mon grand dictionnaire illustré, fait de même pour chaud(e) et normal(e), en précisant les deux formes du pluriel pour le dernier ( "normal(e) [...] adj.; pluriel : normaux, normales. »), mais pas pour chasseur, qui ne figure qu'au masculin, alors que pour un item de flexion comparable, chanteur, la variation en genre est bien explicitée dans le composant flexionnel («chanteur [...] nom masc. ; fém. chanteuse. »).

${ }^{10}$ Les dictionnaires pour le cycle 2 offrent environ cinq fois moins de paradigmes de verbes modèles que ceux pour le cycle 3 (respectivement 13 contre 56 pour les dictionnaires Hachette, 16 contre 62 pour les Robert et 12 contre 79 pour les Larousse (Larousse des débutants vs Larousse junior)) et le nombre de temps et de modes représentés y est également plus réduit (indicatif présent, futur, imparfait et passé composé, et impératif présent dans le Hachette benjamin, auxquels s'ajoutent les participes passé et présent dans le Larousse des débutants, et les conditionnel et subjonctif présents dans le Robert benjamin), mais, alors que les paradigmes de conjugaison sont complets dans les dictionnaires pour le cycle 2, ils ne présentent que des sélections de formes dans ceux pour le cycle 3 .

${ }^{11}$ La place faite à la réforme de l'orthographe de 1990 reste à évaluer, mais elle est actuellement prise en compte par les dictionnaires juniors des trois éditeurs majeurs : 


\begin{abstract}
- en 2008, le seul ouvrage sur le marché qui indiquait explicitement prendre en compte cette réforme était la nouvelle édition du Larousse junior, qui lui consacrait un paratexte (pp. IX-X) et des remarques (comme s.v. événement : «La nouvelle orthographe permet d'écrire aussi évènement, avec un accent grave sur le deuxième e. » ou s.v. goût : «La nouvelle orthographe permet d'écrire aussi gout, sans accent circonflexe. ») qui étaient introduites par la même formule et qui étaient présentées dans l'avant-propos (p. VII : «En fin d'article, des remarques (précédées d'une puce rouge) signalent les difficultés de langue $[. .$.$] Ces remarques incluent systématiquement les recommandations de la$ réforme de l'orthographe de 1990 (voir La nouvelle orthographe pp. IX-X). » ;

- en 2010, le Dictionnaire Hachette junior a enrichi sa préface en indiquant que « [1] es rectifications de l'orthographe, conformément aux programmes, sont systématiquement indiquées et présentées sous la forme de variantes à la fin des entrées » et il a introduit des remarques orthographiques en fin d'articles (comme s.v. évènement : "On écrit aussi événement. » ou s.v. goût : «On écrit aussi gout. ») qui présentent soit la forme ancienne quand l'orthographe réformée est présentée en adresse, soit la forme réformée ;

- et en 2013, Le Robert junior prend en compte lui aussi la réforme dans les articles par le biais de remarques (par exemple $s . v$. évènement ou événement : «Écrire évènement avec un accent grave sur le deuxième $e$ est conforme à la nouvelle orthographe », mais sans mentionner la graphie gout s.v. goût) et il introduit explicitement cette évolution dans son avant-propos (qui indique : "L'orthographe tient compte des recommandations proposées en 1990, approuvées par l'Académie française, afin de former les élèves du cycle 3 à «l'orthographe réformée [qui] est la référence » (B.O. $n^{\circ} 3,19$ juin 2008). L'écriture de certains mots évolue vers plus de simplicité et de logique (handball en un seul mot sur le modèle de football, évènement accentué comme avènement, pizzéria francisé à la manière de cafétéria, millepatte à rapprocher de millefeuille...). Les nouvelles graphies qui figurent en entrée principale sont employées dans l'ensemble du dictionnaire ; d'autres sont simplement signalées en remarque dans l'article. Elles ne peuvent être considérées comme fautives, notamment dans le cadre scolaire. », p. X).
\end{abstract}

${ }^{12}$ L'existence, depuis 2001, d'un Dictionnaire des mots difficiles à lire destiné aux élèves de l'école élémentaire et comportant un millier d'articles donne une certaine vue du lexique dont la prononciation ne se déduit pas aisément de la forme graphique.

${ }^{13}$ «Bœuf se prononce [bœf], mais au pluriel on prononce [bø] : regarde page 12. » / « Prononciation [bœf], pluriel [bø]. ».

${ }^{14}$ «Eil se prononce [œj]. Au pluriel yeux se prononce [jø] : regarde page 12. ».

${ }^{15}$ À la différence de ce qu'il en est pour les graphies (cf. $\S 2.1 .1$ ), les tableaux de conjugaison ne sont d'aucun secours concernant la prononciation des formes fléchies des verbes.

${ }^{16}$ S.v. zoom dans le Dictionnaire Hachette junior : «Zoom est un mot anglais : on prononce [zum]. ».

17 «Un éboueur est un homme qui ramasse les ordures dans les rues. ».

18 «Les aveugles sont des personnes qui ne peuvent pas voir. » / Les sourds sont des personnes qui ne peuvent pas entendre. » / « Des écailles sont des petites plaques dures qui recouvrent le corps de certains animaux. ».

${ }^{19}$ «Les écailles, ce sont les petites plaques dures qui recouvrent le corps des poissons, des serpents, des lézards, des tortues. ».

${ }^{20} \mathrm{La}$ contextualisation peut aussi ne fournir aucune indication flexionnelle, soit qu'une partie de celles-ci figure en remarque (ex. : le pluriel s.v. final), soit que l'article n'en comporte aucune (ex. : prochain, inévitable) :

- s.v. final : «La dictée est terminée : mettez le point final. » (en remarque : «L'instant final, les résultats finaux. »); - s.v. prochain : «Nous descendons au prochain arrêt. » (pas de remarque);

- s.v. inévitable : «La voiture roulait trop vite, l'accident était inévitable. » (en légende d'iconographie : «Le guépard va arrêter de courir, c'est inévitable. »; pas de remarque).

${ }^{21}$ Les marques diachroniques indiquent si les usages lexicaux sont vieillissants ou actuels.

${ }^{22}$ Les marques diaévaluatives caractérisent les attitudes énonciatives des locuteurs qui emploient les unités lexicales marquées.

${ }^{23}$ Les marques diaphasiques spécifient la formalité plus ou moins grande des usages lexicaux.

${ }^{24}$ Les marques diatextuelles indiquent dans quels types de discours ou genres textuels les unités lexicales sont employées. 


\begin{abstract}
${ }^{25}$ Ce marquage pouvait être renforcé par la mention dans la contextualisation soit d'un titre de texte où cet item est supposé être employé ( Dans "Aladin et la lampe merveilleuse», Aladin conduit la princesse dans sa nouvelle demeure. », s.v. demeure), soit d'un localisateur générique (comme s.v. licorne : «Dans les légendes, la licorne est le symbole de ce qui est innocent et pur et sa corne a des pouvoirs magiques. »).

${ }^{26}$ Le segment de texte entre crochets figure dans les éditions de 2008 et 2013, mais pas dans celle de 2003.

${ }^{27}$ Les marques diamédiales indiquent quel est le "canal” d'expression (oral ou écrit) privilégié pour l'emploi des
\end{abstract} unités lexicales marquées.

${ }^{28}$ Les marques dia-intégratives signalent des mots d'origine étrangère mal intégrés d'une manière ou d'une autre à la langue française, en indiquant les idiomes d'origine. Ces emprunts font alors l'objet de considérations extérieures à celles de la rubrique étymologique des articles.

${ }^{29}$ Dans le Larousse junior de 2003 : «C'est un mot anglais, il vaut mieux dire cadreur, euse. ».

${ }^{30}$ Les marques dianormatives signalent les usages lexicaux déconseillés et expriment donc les recommandations les plus strictement prescriptives.

${ }^{31}$ Dans Le Robert junior illustré jusqu'en 2010 : « On dit plutôt les malades mentaux. ».

${ }^{32}$ Dans Le Robert benjamin jusqu'en 2012 : «Quand on parle avec des gens sérieux, c'est mieux de dire un petit garçon ou une petite fille. ».

${ }^{33}$ De 1993 à 2003, Le Robert junior illustré indiquait s.v. joujou : « Ce mot est utilisé par les très jeunes enfants. ».

${ }^{34}$ Les marques diatopiques situent géographiquement les usages lexicaux, qu'il s'agisse de spatialité intraterritoriale (pour les usages hexagonaux) ou extraterritoriale (pour ceux de la francophonie).

${ }^{35}$ Dans Le Robert junior illustré de 2013, le marquage n'est plus diatextuel mais diaphasique : « Ce mot appartient au langage soutenu ».

${ }^{36}$ «L'emploi de ce verbe est critiqué. On dira : Tout ceci lui est ou le laisse indifférent.» (s.v. indifférer).

37 «On peut prendre le bus ou marcher, cela m'est indifférent. » (Larousse junior, s.v. indifférent, e 1.); «Que tu partes ou que tu restes, cela m'est indifférent. » (Dictionnaire Hachette junior, s.v. indifférent, ente 1.) ; "Que tu partes ou que tu restes, cela m'est indifférent. " (Robert junior illustré, s.v. indifférent, indifférente 2. depuis 2005 , s.v. indifférent 1. de 1993 à 2003) ; «Cela m'est totalement indifférent que tu viennes ou pas. » (Fleurus junior, s.v. indifférent, ente 1.) ; "On va où tu veux, cela m'est indifférent. 》 (Dictionnaire Auzou junior, s.v. indifférent, indifférente 1.); «Que l'on parte à la mer ou à la montagne pour les vacances m'est indifférent. » (Le grand dictionnaire de français, s.v. indifférent(e) 1.).

${ }^{38}$ « Situation dans laquelle on doit choisir entre deux possibilités. Soit elle accepte, soit elle refuse, il n'y a pas d'autre alternative. " (Larousse junior) ; "Choix que l'on doit faire entre deux solutions. Dire ce qu'on pense ou se taire, il n'y a pas d'autre alternative. " (Dictionnaire Hachette junior) ; "Situation dans laquelle on se trouve quand on doit choisir entre deux solutions. L'alternative est claire : soit tu restes ici, soit tu viens avec nous. » (Robert junior illustré) ; "Choix entre deux possibilités. Accepter ou refuser, il n'y a pas d'autre alternative. » (Fleurus junior) ; "Situation dans laquelle on doit choisir entre deux solutions complètement différentes. Le héros n'avait qu'une alternative : fuir ou combattre. " (Dictionnaire Auzou junior), «L'alternative est simple : obéir ou démissionner, le choix à faire entre deux solutions. »(Dictionnaire Maxi débutants).

${ }^{39}$ «Une alternative est un choix entre deux possibilités. On ne peut donc pas « avoir deux alternatives », ni « choisir entre deux alternatives ». Fuir ou se rendre, ils n'avaient qu'une alternative. L'emploi de ce mot au sens de "solution (de rechange) » est critiqué. Ainsi, on évitera de dire : «Je n'avais pas d'autre alternative que partir. »» (s.v. alternative).

${ }^{40} \mathrm{Il}$ est particulièrement révélateur que, si Le Robert junior illustré et le Dictionnaire Maxi débutants mettent en adéquation la contextualisation qu'ils proposent avec l'emploi que préconisent respectivement leur définition et leur glose, les contextualisations des quatre autres dictionnaires évoqués illustreraient mieux, par les restricteurs qu'ils comportent ("pas d'autre alternative », "qu'une alternative »), l'acception réprouvée omise que l'acception normative qu'ils retiennent. Il en va d'ailleurs de même pour la première contextualisation du Bescherelle pratique, qui aurait préférentiellement pu prendre, par exemple, la forme L'alternative qui se présentait à eux était de fuir ou de se rendre. 
${ }^{41}$ «Pallier les inconvénients d'une situation. » (Larousse junior); «Pour s'éclairer, les bougies permettent de pallier la coupure d'électricité. » (Dictionnaire Hachette junior) ; "Léa suit des cours particuliers pour pallier ses insuffisances en mathématiques. » (Le Robert junior illustré depuis 2005 - «Yves » a précédé «Léa» de 1993 à 2003); «Pallier un manque. » (Fleurus junior); "Mon frère pallie ses mauvais résultats par de la bonne volonté. » (Dictionnaire Auzou junior) ; «Il faudrait pallier ces inconvénients par des mesures appropriées » (Dictionnaire Maxi débutants).

${ }^{42}$ « Pallier est suivi d'un complément d'objet direct. » (Le Robert junior illustré) ; « La tournure pallier à est déconseillée. »(Dictionnaire Maxi débutants).

43 «Jessie est allée chez le coiffeur. » (Larousse junior); «Tes cheveux sont trop longs, va chez le coiffeur!» (Dictionnaire Hachette junior) ; "Théo est allé chez le coiffeur.» (s.v. coiffeur, coiffeuse dans Le Robert junior illustré depuis 2005 - «Luc » a précédé « Théo » de 1993 à 2003) ; "Aller chez sa coiffeuse se faire couper les cheveux. " (Fleurus junior); "Je vais chez le coiffeur mardi.» (s.v. coiffeur, coiffeuse dans le Dictionnaire Auzou junior); «Pierre est allé chez le coiffeur» (Dictionnaire Maxi débutants).

${ }^{44}$ Cette contextualisation figure après l'adresse microstructurelle se blesser qui est présentée $s . v$. blesser 1 . De 1993 à 2003, s.v. blesser 1, le prénom de la personne blessée différait ( «Flora s'est blessée à la main ») et il n’y avait pas d'adressage microstructurel.

${ }^{45}$ Cf. Corbin $(2004, \S 2.3 .2$.) qui, dans une modélisation de l'activité lexicographique en trois strates (métalinguistique, discursive et de posttraitement), observe une « intrication [...] du traitement métalinguistique et de sa mise en discours qui rend nécessaire leur discrimination conceptuelle » et pose, en illustrant ce propos au moyen des mêmes exemples, qu'il convient - pour les lexicographes en formation (mais ceci est également vrai pour les utilisateurs des dictionnaires) - de repérer « les messages métalinguistiques sous-jacents aux options discursives retenues ».

${ }^{46}$ Le segment de texte entre crochets ne figure plus dans l'édition de 2013.

${ }^{47}$ Cette injonction a été remplacée dans l'édition de 2013 par une contextualisation non prescriptive : «J'ai ajouté une pincée de sel. ». 